\title{
Synthesis and Cytotoxicity Evaluation of Tropinone Derivatives
}

\author{
Xiu-Juan Yin · Chang-An Geng $\cdot$ Xing-Long Chen • \\ Chang-Li Sun · Tong-Hua Yang $\cdot$ Tian-Ze Li $\cdot$ \\ Jun Zhou $\cdot$ Xue-Mei Zhang $\cdot$ Ji-Jun Chen
}

Received: 19 December 2016/Accepted: 12 March 2017/Published online: 20 March 2017

(C) The Author(s) 2017. This article is an open access publication

\begin{abstract}
Sixteen tropinone derivatives were prepared, and their antitumor activities against five human cancer cells (HL60, A-549, SMMC-7721, MCF-7 and SW480) were evaluated with MTS [3-(4,5-dimethylthiazol-2-yl)-5-(3-carboxy methoxyphenyl)-2-(4-sulfopheny)-2H-tetrazolium] assay. Most of the derivatives exhibited better activities compared with tropinone at the concentration of $40 \mu \mathrm{M}$. Particularly, derivative $\mathbf{6}$ showed significant activities with $\mathrm{IC}_{50}$ values of 3.39, 13.59, 6.65, 13.09 and $12.38 \mu \mathrm{M}$ respectively against HL-60, A-549, SMMC-7721, MCF-7 and SW480 cells, which suggested more potent activities than that of cis-dichlorodiamineplatinum (DDP).
\end{abstract}

X.-J. Yin · C.-A. Geng $\cdot$ X.-L. Chen · C.-L. Sun · T.-H. Yang ·

T.-Z. Li · J. Zhou · X.-M. Zhang · J.-J. Chen $(\bowtie)$

State Key Laboratory of Phytochemistry and Plant Resources in West China, Kunming Institute of Botany, Chinese Academy of Sciences, Kunming 650201, China

e-mail: chenjj@mail.kib.ac.cn

X.-J. Yin · X.-L. Chen

University of Chinese Academy of Sciences, Beijing 100049,

China
C.-A. Geng - T.-H. Yang · T.-Z. Li · X.-M. Zhang · J.-J. Chen

Yunnan Key Laboratory of Natural Medicinal Chemistry,

Kunming Institute of Botany, Chinese Academy of Sciences, Kunming 650201, China 


\section{Graphical Abstract}

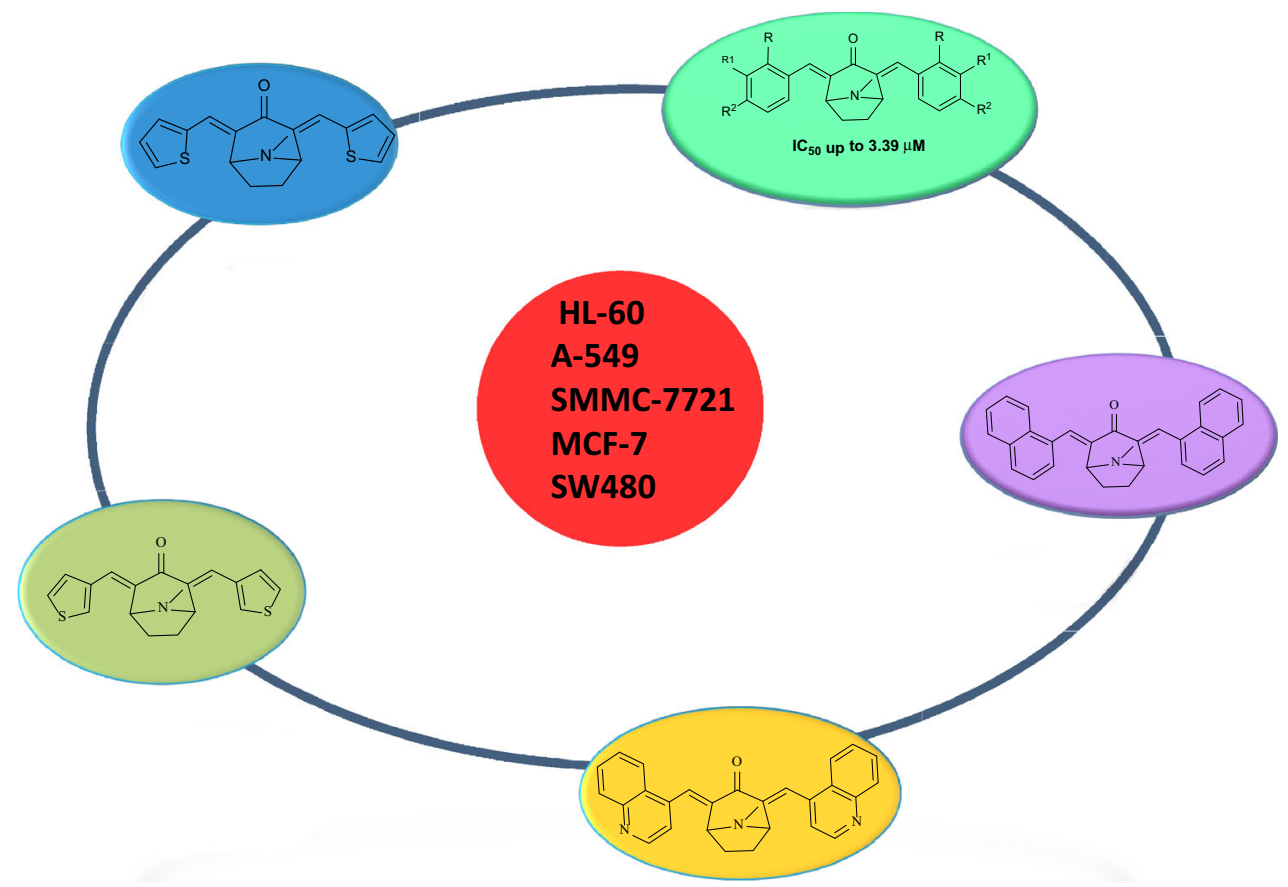

Keywords Tropinone $\cdot$ Claisen-Schmidt reaction $\cdot$ MTS $\cdot$ Cytotoxicity

\section{Introduction}

Cancer, a diverse group of diseases characterized by those uncontrolled growth of tumor cells, is a leading cause of morbidity and mortality globally, which brings heavy economic burden to society and individuals $[1,2]$. The conquest of cancer continued to pose great challenges to medical science since the pathogenesis is complicated and yet not well clarified [3-5]. With the understanding of cancer pathophysiology, some breakthrough therapies for the treatment of cancer were developed [6], but exploring novel types of antitumor drug is still needed.

Among the natural products studied in the 19th and early 20th centuries, tropane alkaloids attracted particular interest due to their potent and extensive biological activities [7], including regulating the secretion of monoamine neurotransmitter [8-11], glycine receptor [12], and acetylcholine receptor [13-15]. The structural scaffold of tropane is a bicyclic amine with a pyrrolidine and piperidine ring sharing a nitrogen atom and two carbon atoms. Tropinone as a natural tropane alkaloid mainly distributed in Solanaceae plants (Cyphomandra betacea) [16], and the total synthesis of tropinone in 1917 by Sir Robert Robinson represented a landmark achievement in organic synthesis [17]. $\alpha, \beta$-Unsaturated ketone is a kind of important organic intermediate, which is widely used in the fields of medicine, chemistry, material science, biology and so on. Recent studies suggested that chalcones [18-20] and $\alpha, \beta$ unsaturated ketones (including coumarin [21], pyrimidine [22], thiosemicarbazide [23], imidazole [24], piperidine [25] analogues) had antitumor activities. Therefore, it is speculated that $\alpha, \beta$ unsaturated ketones with the structural scaffold of tropane may have antitumor activities. Thus we designed and synthesized a series of tropinone derivatives, and their preliminary biological evaluation was performed for their inhibitory activities in five human cancer cell lines (HL-60, A-549, SMMC-7721, MCF-7 and SW480) using MTS [3-(4, 5-dimethylthiazol-2-yl)-5-(3-carboxymethoxyphenyl)-2(4-sulfopheny)-2H-tetrazolium] method. The pattern of apoptosis in vitro against cancer depended upon cell line and dose of the compound [26, 27] and the dose was established according to the related literatures [28-30].

\section{Results and Discussion}

\subsection{Chemistry}

With an objective to obtain target compounds which have activities against five human cancer cells in vitro, tropinone was reacted with corresponding benzaldehyde or other aromatic aldehydes by Claisen-Schmidt condensation in the mixed solution of a catalytic amount of $\mathrm{NaOH}$, and ethanol to 


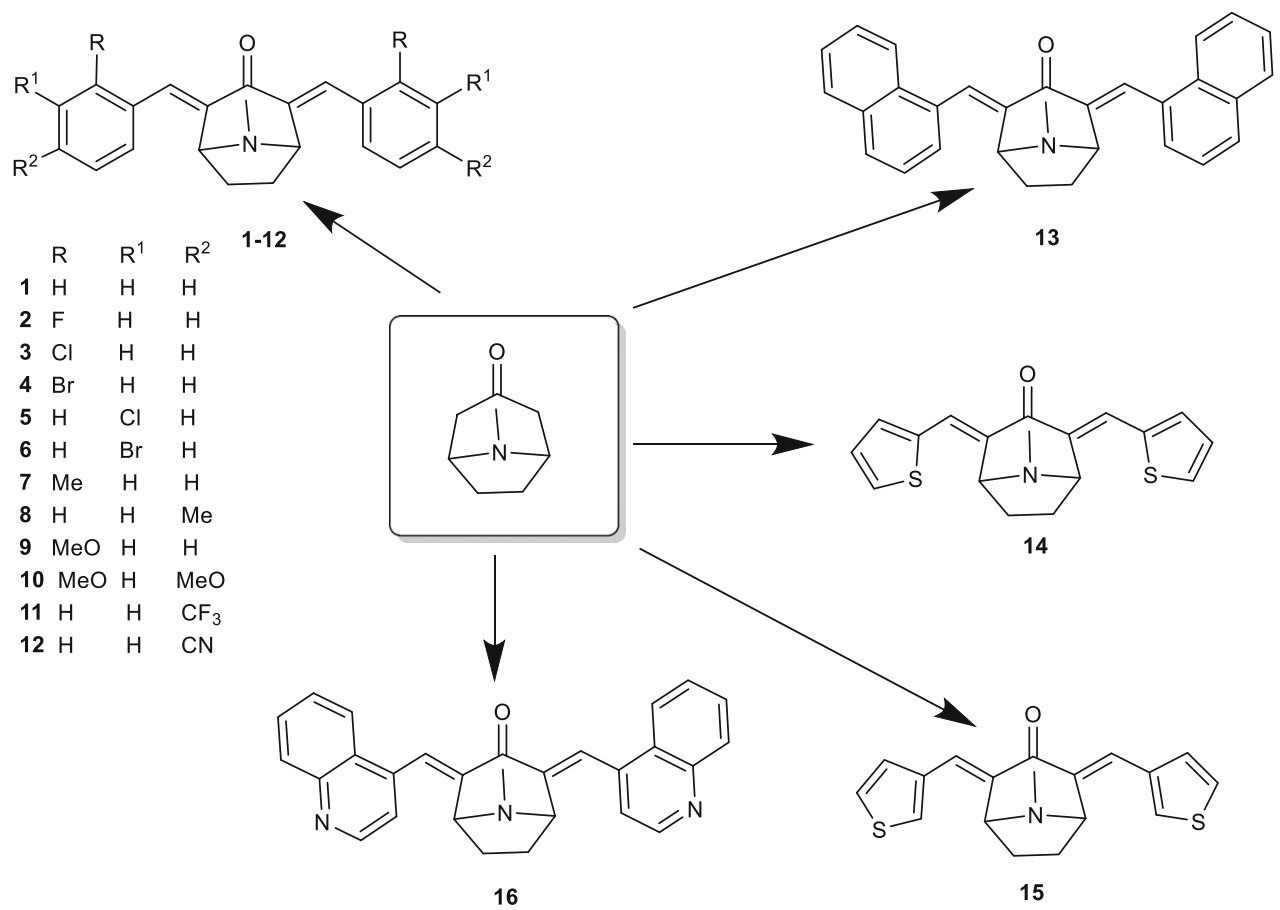

Reagents and conditions: corresponding aldehydes, $\mathrm{NaOH}, \mathrm{EtOH}, 0{ }^{\circ} \mathrm{C} \sim \mathrm{r}$. t., $70-90 \%$

Fig. 1 Synthesis of the tropinone derivatives

generate compounds 1-16. The results suggested that introduction of 2,4-bis(phenylmethylene) groups improved activities compared with that of tropinone (Fig. 1).

\subsection{Biological Evaluation}

Derivatives $\mathbf{2}$ and $\mathbf{8}$ were synthesized [31-34] and evaluated against Molt 4/C8, CEM cells and L1210 cells in vitro, which were effective indicators of derivatives having potential clinical utility. Derivatives 1-16 were tested for antitumor activities against five human cancer cells (HL60, A-549, SMMC-7721, MCF-7 and SW480) by MTS method (Tables 1, 2).

Derivative 1 displayed potential inhibitory activity against HL-60 cell, which was very similar with that of derivative 9, and their inhibitory rates were up to $88.30 \pm 3.33 \%$ and $81.56 \pm 1.98 \%$ at the concentration of $40 \mu \mathrm{M}$, respectively. Derivatives 1 and 9 with unsubstituted and ortho- methoxyl substituted patterns at the phenyl ring, displayed inhibitory potency against HL-60 cell with the $\mathrm{IC}_{50}$ values of 13.62 and $18.97 \mu \mathrm{M}$, respectively. Compared with the tropinone, the inhibitory activities of derivative $6 \quad\left(\mathrm{IC}_{50}=3.39 \mu \mathrm{M}\right)$ showed a 3-9 fold enhancement and was better than that of the positive DDP. Derivatives 5, 10 and 14 possessed higher inhibitory activities than that of tropinone with the inhibitory rates of $55.69 \pm 2.59,59.19 \pm 1.64$ and $71.16 \pm 2.43 \%$ at the concentration of $40 \mu \mathrm{M}$, respectively.
Derivatives 1 and $\mathbf{6}$ showed significantly inhibitory activities against A-549 cell with $\mathrm{IC}_{50}$ values of 16.78 and $13.59 \mu \mathrm{M}$, respectively. Derivatives 4, 9, 10 and 15 possessed moderate activities with inhibitory rates of $57.58 \pm 2.99,67.17 \pm 2.80$, $55.93 \pm 0.96$ and $54.41 \pm 2.93 \%$ at the concentration of $40 \mu \mathrm{M}$, respectively.

Derivatives $\mathbf{1}$ and $\mathbf{9}$ displayed inhibitory potency against SMMC-7721 cell with $\mathrm{IC}_{50}$ values of 14.24 and $28.90 \mu \mathrm{M}$, respectively. Derivative $6\left(\mathrm{IC}_{50}=6.65 \mu \mathrm{M}\right)$ showed significant activity against SMMC-7721 cell, which exhibited more potential than that of DDP $\left(\mathrm{IC}_{50}=13.86 \mu \mathrm{M}\right)$.

Derivatives $1,4,6,9$ and 16 possessed activities against MCF-7 cell with inhibitory rates of $69.77 \pm 1.70,51.95 \pm$ $0.22,68.19 \pm 1.38,72.91 \pm 0.12$ and $60.74 \pm 2.60 \%$ at the concentration of $40 \mu \mathrm{M}$, respectively. Particularly, Derivatives $\mathbf{1}, \mathbf{6}$ and 9 showed significant activities with $\mathrm{IC}_{50}$ values of $16.57,13.09$ and $16.31 \mu \mathrm{M}$, respectively.

Derivatives 4, 5, 9 and 16 demonstrated moderate inhibitory activity against SW480 cell with inhibitory rates of $67.73 \pm 2.01,54.20 \pm 1.30,87.84 \pm 1.86 \%$ and $68.09 \pm$ $1.12 \%$ at the concentration of $40 \mu \mathrm{M}$, respectively.

\subsubsection{The Preliminary Structure-Activity Relationships (SARs)}

SARs were discussed based on the bioassay results against five human cancer cells in vitro (Fig. 2). Derivative 1, with 
Table 1 The inhibitory rates of tropinone derivatives against tumor cells in vitro

\begin{tabular}{|c|c|c|c|c|c|}
\hline \multirow[t]{2}{*}{ Comp. } & \multicolumn{5}{|c|}{ Inhibitory rates $(\%)^{\mathrm{a}}$} \\
\hline & HL-60 & A-549 & SMMC-7721 & MCF-7 & SW480 \\
\hline DDP & $84.33 \pm 0.47$ & $70.07 \pm 1.50$ & $63.57 \pm 3.12$ & $67.28 \pm 1.10$ & $59.00 \pm 2.10$ \\
\hline Taxol & $95.81 \pm 0.17$ & $73.37 \pm 0.28$ & $90.66 \pm 0.56$ & $62.28 \pm 0.86$ & $56.03 \pm 0.31$ \\
\hline Tropinone & $20.96 \pm 2.97$ & $11.38 \pm 1.80$ & $8.65 \pm 1.67$ & $26.98 \pm 2.94$ & $8.68 \pm 2.67$ \\
\hline 1 & $\mathbf{8 8 . 3 0} \pm 3.33$ & $91.99 \pm 0.51$ & $89.16 \pm 3.56$ & $69.77 \pm 1.70$ & $67.55 \pm 0.78$ \\
\hline 2 & $1.32 \pm 2.66$ & $32.07 \pm 1.16$ & $10.62 \pm 1.72$ & $26.05 \pm 0.69$ & $32.23 \pm 1.77$ \\
\hline 3 & $6.51 \pm 2.36$ & $15.35 \pm 2.60$ & $24.96 \pm 0.60$ & $38.99 \pm 2.21$ & $3.84 \pm 4.60$ \\
\hline 4 & $6.63 \pm 2.35$ & $57.58 \pm 2.99$ & $25.17 \pm .2$ & $51.95 \pm 0.22$ & $67.73 \pm 2.01$ \\
\hline 5 & $55.69 \pm 2.59$ & $44.10 \pm 3.90$ & $44.23 \pm 1.94$ & $42.42 \pm 1.75$ & $54.20 \pm 1.30$ \\
\hline 6 & $93.32 \pm 1.22$ & $95.17 \pm 0.50$ & $94.55 \pm 0.48$ & $68.19 \pm 1.38$ & $67.90 \pm 2.42$ \\
\hline 7 & $28.99 \pm 1.20$ & $13.77 \pm 0.19$ & $6.53 \pm 1.98$ & $5.33 \pm 1.49$ & $0.38 \pm 1.64$ \\
\hline 8 & $8.11 \pm 3.06$ & $12.21 \pm 2.15$ & $1.37 \pm 1.10$ & $1.92 \pm 1.48$ & $5.98 \pm 0.91$ \\
\hline 9 & $81.56 \pm 1.98$ & $67.17 \pm 2.80$ & $57.35 \pm 0.40$ & $72.91 \pm 0.12$ & $87.84 \pm 1.86$ \\
\hline 10 & $59.19 \pm 1.64$ & $55.93 \pm 0.96$ & $41.75 \pm 2.55$ & $26.05 \pm 0.54$ & $27.40 \pm 1.46$ \\
\hline 11 & $12.24 \pm 1.59$ & $2.44 \pm 3.11$ & $1.83 \pm 2.85$ & $0.67 \pm 0.81$ & $6.65 \pm 2.27$ \\
\hline 12 & $5.93 \pm 2.30$ & $9.24 \pm 1.86$ & $14.26 \pm 2.84$ & $3.56 \pm 3.93$ & $18.62 \pm 2.98$ \\
\hline 13 & $39.43 \pm 1.47$ & $1.25 \pm 1.35$ & $10.87 \pm 2.88$ & $16.89 \pm 4.58$ & $15.98 \pm 1.84$ \\
\hline 14 & $71.16 \pm 2.43$ & $36.96 \pm 0.43$ & $33.60 \pm 2.70$ & $44.71 \pm 1.90$ & $35.28 \pm 3.17$ \\
\hline 15 & $27.50 \pm 2.36$ & $54.41 \pm 2.93$ & $47.78 \pm 0.55$ & $23.19 \pm 1.63$ & $22.71 \pm 2.21$ \\
\hline 16 & $2.33 \pm 3.08$ & $36.73 \pm 3.16$ & $17.06 \pm 0.17$ & $60.74 \pm 2.60$ & $68.09 \pm 1.12$ \\
\hline
\end{tabular}

Taxol was tested at the concentration of $5 \mu \mathrm{M}$ and other derivatives were tested at the concentration of $40 \mu \mathrm{M}$

${ }^{\text {a }}$ The inhibitory rates expressed as $\overline{\mathrm{X}} \pm \mathrm{SD}(n=3)$

The derivatives of the significance [bold] showed significant activities against HL-60, A-549, SMMC-7721, MCF-7 and SW480 cells

unsubstituted phenylmethylene group, exhibited better activities against five human cancer cells $\left(\mathrm{IC}_{50}=13.62\right.$, $16.78,14.24,16.57$ and 11.95, respectively). Derivatives 2, $\mathbf{3}, \mathbf{4}$ and $\mathbf{7}$ showed less potent activities, suggesting that the 2-halogenated or 2-methylation derivatives were unfavorable for maintaining activities. The chlorinated derivative 5 at meta-position of phenyl ring displayed slightly lower activities than that of bromated derivative $\mathbf{6}$ against five human cancer cells. Derivative $\mathbf{6}$ showed significant activities against HL-60, A-549, SMMC-7721, MCF-7 and SW480 cell $\left(\mathrm{IC}_{50}=3.39,13.59,6.65,13.09\right.$ and 12.38, respectively) and more potent activities than that of DDP. Derivative 9 with a methoxy group at the C-2 position of the phenyl ring indicated higher activities than that of dimethoxy substituted derivative $\mathbf{1 0}$ with 2,4-position at the phenyl ring. Inhibitory activities of derivatives $\mathbf{8}, \mathbf{1 1}$ and 12, with electron-donating group $\left(-\mathrm{CH}_{3}\right)$ or an electronacceptor groups $\left(\mathrm{CF}_{3}, \mathrm{CN}\right)$ at the para- phenyl ring, showed similar activity against five human cancer cells compared with that of tropinone. From the above results, it is suggested that 2,4-bis-phenylmethylene groups were favorable for inhibitory activities. When the 2,4-bisphenylmethylene groups were changed to be 2,4-bis-4heteroaryl-methylene groups, the inhibitory activities
Table 2 The $\mathrm{IC}_{50}(\mu \mathrm{M})$ values of derivatives $\mathbf{1 , 6}$ and 9 against tumor cells in vitro

\begin{tabular}{lccccc}
\hline Comp. & \multicolumn{5}{l}{$\mathrm{IC}_{50}(\mu \mathrm{M})$} \\
\cline { 2 - 6 } & $\mathrm{HL}-60$ & $\mathrm{~A}-549$ & SMMC-7721 & MCF-7 & SW480 \\
\hline DDP & 4.31 & 17.39 & 13.86 & 16.31 & 19.07 \\
Taxol & $<0.008$ & $<0.008$ & $<0.008$ & $<0.008$ & $<0.008$ \\
$\mathbf{1}$ & 13.62 & 16.78 & 14.24 & 16.57 & 11.95 \\
$\mathbf{6}$ & $\mathbf{3 . 3 9}$ & 13.59 & $\mathbf{6 . 6 5}$ & 13.09 & 12.38 \\
& 18.97 & 29.23 & 28.90 & 21.14 & 19.79 \\
\hline
\end{tabular}

Dose-response of antitumor activity was performed in triplicate and monitored with Thermo Scientific Multiskan FC. $\mathrm{IC}_{50}$ values for the derivatives 1, 6, 9 and DDP were determined from the dose-response curves obtained with five concentrations from the range of 0.064 to $40 \mu \mathrm{M}$ against five human cancer cells (HL-60, A-549, SMMC-7721, MCF-7 and SW480), and calculated by the Reed and Muench method [36]. $\mathrm{IC}_{50}$ values for taxol were determined from the dose-response curves obtained with five concentrations from the range of 0.008 to $5 \mu \mathrm{M}$

The derivatives of the significance [bold] showed significant activities against HL-60, A-549, SMMC-7721, MCF-7 and SW480 cells

significantly decreased (derivatives 14-16 compared to 112), while 2,4-bis-naphthylmethylene groups (derivative 13) did not affect the inhibitory activities. 


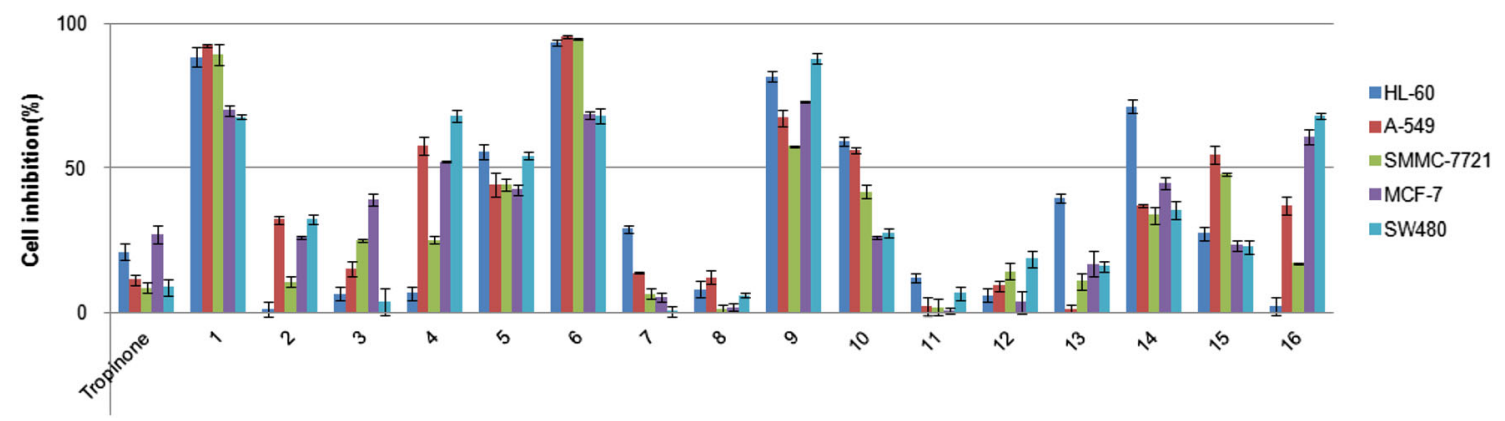

Fig. 2 The inhibitory activities of the tropinone derivatives

\subsection{Conclusion}

In summary, sixteen tropinone derivatives were synthesized and evaluated on HL-60, A-549, SMMC-7721, MCF7 and SW480 cell lines in vitro. Among of them, derivatives $(1,4,5,6,9,10,14,15$ and 16) exhibited higher cytotoxic activities. Particularly, derivatives $\mathbf{1 ,} 6$ and $\mathbf{9}$ had significantly improved activities against HL-60, A-549, SMMC-7721, MCF-7 and SW480 cells with $\mathrm{IC}_{50}$ values of 13.62, 16.78, 14.24, 16.57, $11.95 \mu \mathrm{M}, 3.39,13.59,6.65$, $13.09,12.38 \mu \mathrm{M}$ and $18.97,29.23,28.90,21.14,19.79 \mu \mathrm{M}$ respectively. Structurally, the $\alpha, \beta$-unsaturated ketone moiety was introduced into the tropinone scaffold, which should be responsible for enhanced activities. Among the optimized benzene ring derivatives, derivative $\mathbf{6}$ had higher cytotoxic activities than that of cis-DDP. The SARs of tropinone derivatives provided potential compounds for further investigation on antitumor regents screening.

\section{Experimental Section}

\subsection{Materials and Instruments}

The solvents were dried according to standard procedures. The organic solvents (analytical grade reagents) were purchased from Tianjin Chemical Reagent Co., Ltd (Tianjin, China). The tropinone and corresponding aldehydes were purchased from Alfa Aesar or J\&K Scientific Ltd. ${ }^{1} \mathrm{HNMR}$ and ${ }^{13} \mathrm{CNMR}$ date were recorded in $\mathrm{CDCl}_{3}$ on a $400 \mathrm{MHz}$ spectrometer (Bruker, Bremerhaven, Germany) with the tetramethylsilane (TMS) as the internal standard. Low-resolution mass spectra (MS) and high-resolution mass spectra (HRMS) were measured on Shimadzu liquid chromatography-mass spectrometry (LCMS)-ion trap (IT)-time of flight (TOF) (Shimadzu, Kyoto, Japan). All reaction were carried out under an air atmosphere and monitored by using thinlayer chromatography (TLC, 200-300 mesh, Qingdao Makall Group Co., Ltd; Qingdao, China). Melting points which are uncorrected were determined using a $\mathrm{SGW}^{\circledR} \mathrm{X}-4 \mathrm{~B}$ microscopic melting instrument from Shanghai Precision and Scientific Instrument Co., Ltd (China). The purity of the target compounds was determined by three solvent systems and by HPLC methods.

\subsection{General Procedure for the Synthesis of the Tropinone Derivatives}

Tropinone ( $2 \mathrm{mmol}, 1$ equiv.) and $10 \% \mathrm{NaOH}(5 \mathrm{~mL})$ were dissolved in ethanol $(15 \mathrm{~mL})$. To a solution of corresponding aldehydes ( $4.4 \mathrm{mmol}, 2.2$ equiv.) in ethanol $(5 \mathrm{~mL})$, the mixture was slowly dropped into the above solution at $0{ }^{\circ} \mathrm{C}$ for $20 \mathrm{~min}$. The resulting solution was stirred at room temperature and monitored by TLC. Subsequently, the reaction mixture was neutralized with a solution of $5 \% \mathrm{HCl}$, extracted with EtOAc $(3 \times 30 \mathrm{~mL})$ and washed with saturated $\mathrm{Na}_{2} \mathrm{CO}_{3}$ and saturated $\mathrm{NaCl}$. The organic layer was dried over anhydrous $\mathrm{Na}_{2} \mathrm{SO}_{4}$ and concentrated to dryness under reduced pressure. Purification by column chromatography on silica gel $\mathrm{Et}_{2} \mathrm{NH} /$ $\mathrm{MeOH} / \mathrm{CHCl}_{3}(2: 6: 92, \mathrm{v} / \mathrm{v} / \mathrm{v})$ to the target derivatives.

\subsubsection{8-Methyl-2,4-bis(phenylmethylene)-8-aza- bicyclo[3.2.1]octan-3-one (1)}

Yellow powder, mp 137.7-139.6 ${ }^{\circ} \mathrm{C}$; yield 82\%. ${ }^{1} \mathrm{H}$ NMR $\left(400 \mathrm{MHz}, \mathrm{CDCl}_{3}\right) \delta: 7.84$ (s, 2H, H-9, 10), 7.45-7.26 (m, $10 \mathrm{H}, \mathrm{Ar}$ ), 4.40 (m, 2H, H-1, 5), 2.62-2.60 (m, 2H, H-6, 7), 2.30 (s, 3H, H-8), 2.04-2.03 (m, 2H, H-6, 7). ${ }^{13} \mathrm{C}$ NMR $\left(100 \mathrm{MHz}, \mathrm{CDCl}_{3}\right) \delta: 188.0$ (s, C-3), 138.3 (s, C-2,4), 136.6 (d, C-9, 10), 135.1 (s, C-1', 1" $), 130.2$ (d, C-4', 4"), 128.8 (d, C-2', 2", 6', 6") 128.5 (d, C-3', 3", 5', 5"), 60.8 (d, C-1, 5), 35.8 (s, C-8), 30.3 (t, C-6, 7). IR (KBr) v: 3439, 2950, 1670, 1608, 1584, 1445, 1237, 1164, 946, 778, $692 \mathrm{~cm}-1$. ESIMS: $\mathrm{m} / z, 316[\mathrm{M}+\mathrm{H}]^{+}$, HRESIMS: calc for $\mathrm{C}_{22} \mathrm{H}_{22} \mathrm{NO}[\mathrm{M}+\mathrm{H}]^{+}$316.1696, found 316.1669.

\subsubsection{8-Methyl-2,4-bis(2'-fluoro-phenylmethylene)-8-aza- bicyclo[3.2.1]octan-3-one (2)}

Yellow lamellar crystals $\left(\mathrm{CHCl}_{3}: \mathrm{EtOH}, 80: 20, \mathrm{v} / \mathrm{v}\right), \mathrm{mp}$ 155.9-156.0 ${ }^{\circ} \mathrm{C}$; yield $86 \%,{ }^{1} \mathrm{H}$ NMR $\left(400 \mathrm{MHz}, \mathrm{CDCl}_{3}\right)$ 
$\delta: 7.84$ (s, 2H, H-9,10), 7.38-7.10 (m, 8H, Ar-H), 4.22 (m, 2H, H-1, 5), 2.54-2.51 (m, 2H, H-6, 7), 2.30 (s, 3H, H-8), 2.01-1.97 (m, 2H, H-6, 7). ${ }^{13} \mathrm{C}$ NMR (100 MHz, $\left.\mathrm{CDCl}_{3}\right) \delta$ : 188.0 (s, C-3), 162.1 (d, C-2'), 159.6 (s, C-2" ), 140.2 (s, C-2,4), 130.9 (d, C-9, 10), 130.8 (d, C-4', 4") 129.2 (d, C-6', 6" $), 123.9$ (s, C-4', 4") 123.1 (s, C-1', $\left.1^{\prime \prime}\right), 115.9$ (d, C-3', 3"), 61.1 (d, C-1, 5), 35.4 (s, C-8), 29.9 (t, C-6, 7). IR (KBr) $v: 3439,2943,1672,1612,1589,1435,1218,1056$, 752, $685 \mathrm{~cm}^{-1}$. ESIMS: $\mathrm{m} / z 352[\mathrm{M}+\mathrm{H}]^{+}$, HRESIMS: calc for $\mathrm{C}_{22} \mathrm{H}_{19} \mathrm{NOF}_{2}[\mathrm{M}+\mathrm{H}]^{+}$352.1507, found 352.1502 .

\subsubsection{8-Methyl-2,4-bis(2'-chloro-phenylmethylene)-8-aza- bicyclo[3.2.1]octan-3-one (3)}

Yellow powder, mp 178.0-178.9 ${ }^{\circ} \mathrm{C}$; yield $88 \%,{ }^{1} \mathrm{H}$ NMR $\left(400 \mathrm{MHz}, \mathrm{CDCl}_{3}\right) \delta: 7.92$ (s, 2H, H-9,10), 7.46-7.19 (m, $8 \mathrm{H}, \mathrm{Ar}-\mathrm{H}), 4.14$ (m, 2H, H-1, 5), 2.49-2.47 (m, 2H, H-6, 7), 2.31 (s, 3H, H-8), $1.98-1.96$ (m, $2 \mathrm{H}, \mathrm{H}-6,7) .{ }^{13} \mathrm{C} \mathrm{NMR}$ $\left(100 \mathrm{MHz}, \mathrm{CDCl}_{3}\right) \delta: 188.2$ (s, C-3), 139.8 (s, C-2, 4), 135.1 (s, C-2', 2"), 133.7 (d, C-9, 10), 133.7 (s, C-1', 1"), 130.5 (d, C-3', 3"), 129.9 (d, C-4', 4"), 129.8 (d, C-6', 6"), 126.4 (d, C-5', 5"), 60.6 (d, C-1, 5), 35.2 (s, C-8), 30.1 (t, C-6, 7). IR (KBr) v: 3438, 2941, 1671, 1610, 1586, 1461, 1218, 1059, 753, $662 \mathrm{~cm}^{-1}$. ESIMS: $\mathrm{m} / z, 384[\mathrm{M}+\mathrm{H}]^{+}$, HRESIMS: calc for $\mathrm{C}_{22} \mathrm{H}_{19} \mathrm{NOCl}_{2}[\mathrm{M}+\mathrm{H}]^{+} 384.0916$, found 384.0937 .

\subsubsection{8-Methyl-2,4-bis(2'-bromo-phenylmethylene)-8-aza- bicyclo[3.2.1]octan-3-one (4)}

Yellow powder, mp $157.4-157.8{ }^{\circ} \mathrm{C}$; yield $87 \%,{ }^{1} \mathrm{H}$ NMR $\left(400 \mathrm{MHz}, \mathrm{CDCl}_{3}\right) \delta: 7.92$ (s, 2H, H-9,10), 7.66-7.17 (m, $8 \mathrm{H}, \mathrm{Ar}-\mathrm{H}), 4.14$ (m, 2H, H-1, 5), 2.48-2.47 (m, 2H, H-6, 7), 2.31 (s, 3H, H-8), 1.98-1.96 (m, 2H, H-6, 7). ${ }^{13} \mathrm{C} \mathrm{NMR}$ $\left(100 \mathrm{MHz}, \mathrm{CDCl}_{3}\right) \delta: 188.2$ (s, C-3), 139.7 (s, C-2, 4), 136.0 (d, C-9, 10), 135.5 (s, C-1', $\left.1^{\prime \prime}\right), 135.5$ (s, C-2', 2"), 133.7 (d, C-3', 3"), 133.0 (d, C-5', 5"), 129.9 (d, C-6', 6"), 126.4 (d, C-4', 4"), 60.6 (d, C-1, 5), 35.1 (s, C-8), 30.1 (t, C-6, 7). IR (KBr) v: 3434, 2936, 1667, 1609, 1558, 1477, 1221, 1062, 942, 786, $688 \mathrm{~cm}^{-1}$. ESIMS: $\mathrm{m} / z \quad 471$ $[\mathrm{M}+\mathrm{H}]^{+}$, HRESIMS: calc for $\mathrm{C}_{22} \mathrm{H}_{19} \mathrm{NOBr}_{2}[\mathrm{M}+\mathrm{H}]^{+}$ 471.9906, found 471.9928 .

\subsubsection{8-Methyl-2,4-bis(3'-chloro-phenylmethylene)-8-aza- bicyclo[3.2.1]octan-3-one (5)}

Yellow powder, mp 136.9-137.8 ${ }^{\circ} \mathrm{C}$; yield $86 \%,{ }^{1} \mathrm{H}$ NMR $\left(400 \mathrm{MHz}, \mathrm{CDCl}_{3}\right) \delta: 7.71(\mathrm{~s}, 2 \mathrm{H}, \mathrm{H}-9,10), 7.34-7.21(\mathrm{~m}$, $8 \mathrm{H}, \mathrm{Ar}-\mathrm{H}), 4.30$ (m, 2H, H-1, 5), 2.60-2.58 (m, 2H, H-6, 7), 2.28 (s, 3H, H-8), 2.00-1.96 (m, 2H, H-6, 7). ${ }^{13} \mathrm{C}$ NMR $\left(100 \mathrm{MHz}, \mathrm{CDCl}_{3}\right) \delta: 187.7$ (s, C-3), 142.5 (d, C-9, 10), 139.6 (s, C-2, 4), 137.0 (s, C-3', 3"), 130.3 (d, C-5', 5"),
134.9 (d, C-5', 5"), 129.4 (s, C-2', 2" $), 128.6\left(\mathrm{~d}, \mathrm{C}-4^{\prime}, 4^{\prime \prime}\right)$, 127.1 (d, C-6', 6"), 61.2 (d, C-1, 5), 36.2 (s, C-8), 30.5 (t, C-6, 7). IR (KBr) v: 3437, 2936, 1667, 1609, 1563, 1477, 1221, 1062, 942, 786, $688 \mathrm{~cm}^{-1}$. ESIMS: $\mathrm{m} / \mathrm{z} 384$ $[\mathrm{M}+\mathrm{H}]^{+}$, HRESIMS: calc for $\mathrm{C}_{22} \mathrm{H}_{19} \mathrm{NOCl}_{2}[\mathrm{M}+\mathrm{H}]^{+}$ 384.0916, found 384.0916.

\subsubsection{8-Methyl-2,4-bis(3'-bromo-phenylmethylene)-8-aza- bicyclo[3.2.1]octan-3-one (6)}

Yellow powder, mp 142.4-143.7 ${ }^{\circ} \mathrm{C}$; yield 84\%, ${ }^{1} \mathrm{H}$ NMR $\left(400 \mathrm{MHz}, \mathrm{CDCl}_{3}\right) \delta: 7.72$ (s, 2H, H-9,10), 7.50-7.26 (m, $8 \mathrm{H}, \mathrm{Ar}-\mathrm{H}$ '), 4.32 (m, 2H, H-1, 5), 2.62-2.59 (m, 2H, H-6, 7), 2.30 (s, 3H, H-8), 2.02-1.97 (m, 2H, H-6, 7). ${ }^{13} \mathrm{C}$ NMR $\left(100 \mathrm{MHz}, \mathrm{CDCl}_{3}\right) \delta: 187.4$ (s, C-3), 139.4 (d, C-2, 4), 137.0 (s, C-1', 1" $), 134.9$ (d, C-9, 10), 132.9 (d, C-2', 2"), 131.7 (d, C-4', 4" $), 130.0$ (d, C-5', 5"), 128.6 (d, C-6', 6"), 122.6 (s, C-3', 3"), 60.7 (d, C-1, 5), 35.9 (s, C-8), 30.1 (t, C-6, 7). IR (KBr) v: 3435, 2929, 1668, 1608, 1556, 1470, 1220, 1058, 944, 787, $681 \mathrm{~cm}^{-1}$. ESIMS: $\mathrm{m} / z, 471$ $[\mathrm{M}+\mathrm{H}]^{+}$, HRESIMS: calc for $\mathrm{C}_{22} \mathrm{H}_{19} \mathrm{NOBr}_{2}[\mathrm{M}+\mathrm{H}]^{+}$ 471.9906, found 471.9928 .

\subsubsection{8-Methyl-2,4-bis(2'-methyl-phenylmethylene)-8-aza- bicyclo[3.2.1]octan-3-one (7)}

Yellow powder, mp 151.0-152.2 ${ }^{\circ} \mathrm{C}$; yield 79\%, ${ }^{1} \mathrm{H}$ NMR $\left(400 \mathrm{MHz}, \mathrm{CDCl}_{3}\right) \delta: 7.93$ (s, 2H, H-9,10), 7.27-7.13 (m, $8 \mathrm{H}, \mathrm{Ar}-\mathrm{H}), 4.20(\mathrm{~m}, 2 \mathrm{H}, \mathrm{H}-1,5), 2.51-2.48(\mathrm{~m}, 2 \mathrm{H}, \mathrm{H}-6$, 7), 2.36 (s, 3H, H-8), 2.29 (s, 6H, Me), 2.00-1.98 (m, 2H, $\mathrm{H}-6,7) .{ }^{13} \mathrm{C}$ NMR $\left(100 \mathrm{MHz}, \mathrm{CDCl}_{3}\right) \delta: 188.8$ (s, C-3), 138.8 (s, C-2, 4), 138.0 (s, C-1', 1"), 135.4 (d, C-9, 10), 134.3 (s, C-2', 2"), 130.3 (d, C-4', 4"), $129.2\left(\mathrm{~d}, \mathrm{C}-3^{\prime}, 3^{\prime \prime}\right)$, 128.7 (d, C-6', 6"), 125.6 (s, C-5', 5"), 60.8 (d, C-1, 5), 35.6 (s, C-8), 30.3 (t, C-6, 7), 20.2 (s, $\left.\mathrm{C}_{2^{\prime}}, 2^{\prime \prime}-\mathrm{Me}\right)$. IR (KBr) v: 3439, 2944, 1669, 1612, 1594, 1452, 1205, 1058, 925, $778 \mathrm{~cm}^{-1}$. ESIMS: $\mathrm{m} / \mathrm{z} 344[\mathrm{M}+\mathrm{H}]^{+}$, HRESIMS: calc for $\mathrm{C}_{24} \mathrm{H}_{25} \mathrm{NO}[\mathrm{M}+\mathrm{H}]^{+}$344.2009, found 344.1993.

\subsubsection{8-Methyl-2,4-bis(4'-methyl-phenylmethylene)-8-aza- bicyclo[3.2.1]octan-3-one (8)}

Yellow powder, mp $165.5-166.5{ }^{\circ} \mathrm{C}$; yield $78 \%,{ }^{1} \mathrm{H}$ NMR $\left(400 \mathrm{MHz}, \mathrm{CDCl}_{3}\right) \delta: 7.83(\mathrm{~s}, 2 \mathrm{H}, \mathrm{H}-9,10), 7.54(\mathrm{~d}$, $\mathrm{J}=7.9 \mathrm{~Hz}, 4 \mathrm{H}, \mathrm{Ar}-\mathrm{H}), 7.23(\mathrm{~d}, J=7.9 \mathrm{~Hz}, 4 \mathrm{H}, \mathrm{Ar}-\mathrm{H})$, 4.40 (m, 2H, H-1, 5), 2.63-2.60 (m, 2H, H-6, 7), 2.38 (s, $3 \mathrm{H}, \mathrm{H}-8), 2.30$ (s, 6H, Me), $2.04-1.99$ (m, 2H, H-6, 7). ${ }^{13} \mathrm{C}$ NMR $\left(100 \mathrm{MHz}, \mathrm{CDCl}_{3}\right) \delta: 187.8(\mathrm{~s}, \mathrm{C}-3), 139.2(\mathrm{~s}, \mathrm{C}-2$, 4), 137.5 (s, C-1', 1"), 136.7 (d, C-9, 10), 132.2 (s, C-4', $\left.4^{\prime \prime}\right), 130.4\left(\mathrm{~d}, \mathrm{C}-3^{\prime}, 3^{\prime \prime}, 5^{\prime}, 5^{\prime \prime}\right), 129.3\left(\mathrm{~d}, \mathrm{C}-2^{\prime}, 2^{\prime \prime}, 6^{\prime}, 6^{\prime \prime}\right)$, 60.9 (d, C-1, 5), 35.7 (s, C-8), 30.3 (t, C-6, 7), 21.4 (s, C $4^{\prime}$, $4^{\prime \prime}-\mathrm{Me}$ ). IR (KBr) v: 3438, 2942, 1666, 1599, 1580, 1447, 1239, 1057, 935, $812 \mathrm{~cm}^{-1}$. ESIMS: $\mathrm{m} / z, 344[\mathrm{M}+\mathrm{H}]^{+}$, 
HRESIMS: calc for $\mathrm{C}_{24} \mathrm{H}_{25} \mathrm{NO}[\mathrm{M}+\mathrm{H}]^{+} 344.2009$, found 344.1993.

\subsubsection{8-Methyl-2,4-bis(2'-methoxyl-phenylmethylene)-8- aza-bicyclo[3.2.1] octan-3-one (9)}

Yellow lamellar crystals $\left(\mathrm{CHCl}_{3}: \mathrm{EtOH}, 80: 20, \mathrm{v} / \mathrm{v}\right), \mathrm{mp}$ 155.5-155.7 ${ }^{\circ} \mathrm{C}$; yield $70 \%,{ }^{1} \mathrm{H}$ NMR $\left(400 \mathrm{MHz}, \mathrm{CDCl}_{3}\right.$ ) $\delta: 8.02$ (s, 2H, H-9,10), 7.35-6.90 (m, 8H, Ar-H), 4.27 (m, $2 \mathrm{H}, \mathrm{H}-1,5), 3.82$ (s, 6H, OMe), 2.53-2.51 (m, 2H, H-6, 7), 2.30 (s, 3H, H-8), 2.02-1.99 (m, 2H, H-6, 7). ${ }^{13} \mathrm{C}$ NMR $\left(100 \mathrm{MHz}, \mathrm{CDCl}_{3}\right) \delta: 188.5$ (s, C-3), $158.4\left(\mathrm{~s}, \mathrm{C}-2^{\prime}, 2^{\prime \prime}\right)$, 138.2 (s, C-2, 4), 132.5 (d, C-9, 10), 130.5 (d, C-4', 4"), $130.5\left(\mathrm{~d}, \mathrm{C}-6^{\prime}, 6^{\prime \prime}\right), 124.3\left(\mathrm{~s}, 1^{\prime}, 1^{\prime \prime}\right), 120.0\left(\mathrm{~d}, \mathrm{C}-5^{\prime}, 5^{\prime \prime}\right)$,

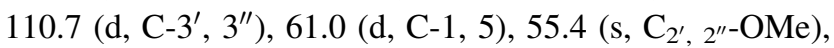
35.2 (s, C-8), 30.3 (t, C-6, 7). IR (KBr) v: 3441, 2944, 1673, 1598, 1486, 1462, 1250, 1058, $757 \mathrm{~cm}^{-1}$. ESIMS: $\mathrm{m} /$ z $376[\mathrm{M}+\mathrm{H}]^{+}$, HRESIMS: calc for $\mathrm{C}_{24} \mathrm{H}_{25} \mathrm{NO}_{3}$ $[\mathrm{M}+\mathrm{H}]^{+}$376.1907, found 376.1906.

\subsubsection{8-Methyl-2,4-bis $\left(2^{\prime}, 4^{\prime}\right.$-dimethoxy- phenylmethylene)-8-aza-bicyclo[3.2.1]octan-3-one (10)}

Yellow oil, yield $79 \%,{ }^{1} \mathrm{H}$ NMR $\left(400 \mathrm{MHz}, \mathrm{CDCl}_{3}\right) \delta: 7.95$ (s, 2H, H-9,10), 7.10-6.41 (m, 6H, Ar-H), 4.23 (m, 2H, $\mathrm{H}-1,5), 3.78$ (s, 12H, OMe), 2.53-2.49 (m, 2H, H-6, 7), 2.34 (s, 3H, H-8), 1.94-1.84 (m, 2H, H-6, 7). ${ }^{13} \mathrm{C}$ NMR $\left(100 \mathrm{MHz}, \mathrm{CDCl}_{3}\right) \delta: 188.0$ (s, C-3), 161.7 (s, C-4', 4"), 159.8 (s, C-2', 2"), 136.3 (s, C-2, 4), 132.2 (d, C-9, 10), 131.1 (d, C-6', 6"), 117.1 (s, C-1', 1"1), 104.1 (s, C-5', 5"),

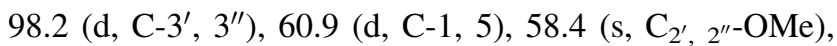
55.2 ( $\left.\mathrm{s}, \mathrm{C}_{4^{\prime}}, 4^{\prime \prime}-\mathrm{OMe}\right), 35.0$ (s, C-8), 30.3 (t, C-6, 7). IR (KBr) $v: 3438.45,2941.52,1677.93,1603.77,1502.15$, 1463.85, 1245.95, 1059.72, $810.43 \mathrm{~cm}^{-1}$. ESIMS: $\mathrm{m} / z 436$ $[\mathrm{M}+\mathrm{H}]^{+}$, HRESIMS: calc for $\mathrm{C}_{26} \mathrm{H}_{29} \mathrm{NO}_{5}[\mathrm{M}+\mathrm{H}]^{+}$ 436.2118, found 436.2128 .

\subsubsection{8-Methyl-2,4-bis(4'-trifluoromethyl- phenylmethylene)-8-aza-bicyclo[3.2.1]octan-3-one (11)}

Yellow lamellar crystals $\left(\mathrm{CHCl}_{3}: \mathrm{EtOH}, 80: 20, \mathrm{v} / \mathrm{v}\right), \mathrm{mp}$ 172.8-173.2 ${ }^{\circ} \mathrm{C}$; yield $88 \%,{ }^{1} \mathrm{H}$ NMR $\left(400 \mathrm{MHz}, \mathrm{CDCl}_{3}\right)$ $\delta: 7.81(\mathrm{~s}, 2 \mathrm{H}, \mathrm{H}-9,10), 7.68(\mathrm{~d}, J=7.8 \mathrm{~Hz}, 4 \mathrm{H}, \mathrm{Ar}-\mathrm{H})$, $7.48(\mathrm{~d}, \mathrm{~J}=7.8 \mathrm{~Hz}, 4 \mathrm{H}, \mathrm{Ar}-\mathrm{H}), 4.33(\mathrm{~m}, 2 \mathrm{H}, \mathrm{H}-1,5)$, 2.64-2.61 (m, 2H, H-6, 7), 2.30 (s, 3H, H-8), 2.05-2.02 (m, $2 \mathrm{H}, \mathrm{H}-6,7) .{ }^{13} \mathrm{C}$ NMR $\left(100 \mathrm{MHz}, \mathrm{CDCl}_{3}\right) \delta: 187.4(\mathrm{~s}$, C-3), 140.1 (s, C-2, 4), 138.4 (s, C-1', $1^{\prime \prime}$ ), 134.8 (d, C-9, 10), 130.3 (d, C-4', 4"), 130.2 (d, C-3', 3" , 5', 5") 125.5 (d, $\left.\mathrm{C}-2^{\prime}, 2^{\prime \prime}, 6^{\prime}, 6^{\prime \prime}\right), 125.5\left(\mathrm{~s}, \mathrm{C}_{4^{\prime}}, 4^{\prime \prime}-\mathrm{CF}_{3}\right), 60.8$ (d, C-1, 5), 35.9 (s, C-8), 30.1 (t, C-6, 7). IR (KBr) v: 3440, 2935, 1672,
1609, 1587, 1411, 1244, 1065, 929, $842 \mathrm{~cm}^{-1}$. ESIMS: $\mathrm{m} / \mathrm{z}$ $452[\mathrm{M}+\mathrm{H}]^{+}$, HRESIMS: calc for $\mathrm{C}_{24} \mathrm{H}_{19} \mathrm{NOF}_{6}$ $[\mathrm{M}+\mathrm{H}]^{+}$452.1444, found 452.1450 .

\subsubsection{8-Methyl-2,4-bis(4'-cyano-phenylmethylene)-8-aza- bicyclo[3.2.1]octan-3-one (12)}

Yellow powder, mp 249.3-249.8 ${ }^{\circ} \mathrm{C}$; yield $79 \%,{ }^{1} \mathrm{H}$ NMR $\left(400 \mathrm{MHz}, \mathrm{CDCl}_{3}\right) \delta: 8.08$ (s, 2H, H-9,10), 7.81-7.70 (m, $4 \mathrm{H}, \mathrm{Ar}-\mathrm{H}), 7.46-7.42$ (m, 4H, Ar-H), 4.28 (m, 2H, H-1, 5), 2.63-2.60 (m, 2H, H-6, 7), 2.29 (s, 3H, H-8), 2.01-1.98 (m, $2 \mathrm{H}, \mathrm{H}-6,7) .{ }^{13} \mathrm{C}$ NMR (100 MHz, $\left.\mathrm{CDCl}_{3}\right) \delta: 187.1$ (s, C-3), 140.7 (s, C-2, 4), 139.4 (s, C-1', 1") 134.2 (d, C-9, 10), 132.3 (d, C-3', 3", 5', 5"), 130.5 (d, C-2', 2" $6^{\prime}, 6^{\prime \prime}$ ), 118.4 (s, C-4', 4"), 112.3 (s, C $\left.4^{\prime}, 4^{\prime \prime}-\mathrm{CN}\right), 60.8(\mathrm{~d}, \mathrm{C}-1,5)$, 36.0 (s, C-8), 29.9 (t, C-6, 7). IR (KBr) v: 3431, 2942, 2227, 1670, 1605, 1585, 1501, 1241, 1060, 944, $835 \mathrm{~cm}^{-1}$. ESIMS: $m / z \quad 366[\mathrm{M}+\mathrm{H}]^{+}$, HRESIMS: calc for $\mathrm{C}_{24} \mathrm{H}_{19} \mathrm{~N}_{3} \mathrm{O}[\mathrm{M}+\mathrm{H}]^{+}$366.1601, found 366.1585.

\subsubsection{8-Methyl-2,4-bis(2'-naphthylmethylene)-8-aza- bicyclo[3.2.1]octan-3-one (13)}

Yellow powder, mp 257.6-258.1 ${ }^{\circ} \mathrm{C}$; yield 78\%, ${ }^{1} \mathrm{H}$ NMR $\left(400 \mathrm{MHz}, \mathrm{CDCl}_{3}\right) \delta: 8.02$ (s, 2H, H-9,10), 7.90-7.86 (m, $8 \mathrm{H}, \mathrm{Ar}-\mathrm{H}), 7.54-7.52(\mathrm{~m}, 6 \mathrm{H}$, naphthalene-H), 4.54-4.53 (m, 2H, H-1, 5), 2.73-2.70 (m, 2H, H-6, 7), 2.48 (s, 3H, H-8), 2.19-2.17 (m, 2H, H-6, 7). ${ }^{13} \mathrm{C}$ NMR (100 MHz, $\mathrm{CDCl}_{3}$ ) $\delta: 188.0$ (s, C-3), 138.7 (s, C-2, 4), 136.7 (d, C-9, 10), 133.2 (s, C-1' $1^{\prime \prime}$ ), 133.1 (s, C-10', 10") 132.7 (s, C-9', $\left.9^{\prime \prime}\right), 130.3\left(\mathrm{~d}, \mathrm{C}-5^{\prime}, 5^{\prime \prime}\right), 128.5$ (d, C-4', 4") 128.2 (d, C-3', $\left.3^{\prime \prime}\right), 127.7$ (d, C-7', 7"), 127.4 (d, C-6', 6" $), 127.0$ (d, C-8', $\left.8^{\prime \prime}\right), 126.6\left(\mathrm{~d}, \mathrm{C}-2^{\prime}, 2^{\prime \prime}\right), 61.1$ (d, C-1, 5), 35.9 (s, C-8), 30.4 (t, C-6, 7). IR (KBr) v: 3442, 2947, 1670, 1612, 1586, 1440, 1207, 1153, 1056, 940, 822, $747 \mathrm{~cm}^{-1}$. ESIMS: $\mathrm{m} / \mathrm{z}$ $416[\mathrm{M}+\mathrm{H}]^{+}$, HRESIMS: calc for $\mathrm{C}_{30} \mathrm{H}_{25} \mathrm{NO}[\mathrm{M}+\mathrm{H}]^{+}$ 416.2009, found 416.1996.

\subsubsection{8-Methyl-2,4-bis(2'-thienylmethylene)-8-aza- bicyclo[3.2.1]octan-3-one (14)}

Yellow powder, mp 163.6-164.5 ${ }^{\circ} \mathrm{C}$; yield $74 \%,{ }^{1} \mathrm{H}$ NMR $\left(400 \mathrm{MHz}, \mathrm{CDCl}_{3}\right) \delta: 7.92$ (s, 2H, H-9,10), 7.52-7.11 (m, $6 \mathrm{H}$, thiophene- $\mathrm{H}), 4.65(\mathrm{~m}, 2 \mathrm{H}, \mathrm{H}-1,5), 2.61-2.58(\mathrm{~m}, 2 \mathrm{H}$, $\mathrm{H}-6,7), 2.42$ (s, 3H, H-8), $1.84-1.79$ (m, 2H, H-6, 7). ${ }^{13} \mathrm{C}$ NMR $\left(100 \mathrm{MHz}, \mathrm{CDCl}_{3}\right) \delta: 186.6$ (s, C-3), 138.3 (s, C-2, 4), 135.6 (s, C-2', $\left.2^{\prime \prime}\right), 133.2$ (d, C-9, 10), 130.1 (d, C-5', $\left.5^{\prime \prime}\right), 128.8\left(\mathrm{~d}, \mathrm{C}-3^{\prime}, 3^{\prime \prime}\right), 127.9\left(\mathrm{~d}, \mathrm{C}-4^{\prime}, 4^{\prime \prime}\right), 61.2(\mathrm{~d}, \mathrm{C}-1,5)$, 36.2 (s, C-8), 29.7 (t, C-6, 7). IR (KBr) v: 3437, 2944, $1655,1589,1450,1417,1239,1166,1040,932,853$, $706 \mathrm{~cm}^{-1}$. ESIMS: $\mathrm{m} / \mathrm{z}, 328[\mathrm{M}+\mathrm{H}]^{+}$, HRESIMS: calc for $\mathrm{C}_{18} \mathrm{H}_{17} \mathrm{NOS}_{2}[\mathrm{M}+\mathrm{H}]^{+}$328.0824, found 328.0808. 
3.2.15 8-Methyl-2,4-bis(3'-thienylmethylene)-8-azabicyclo[3.2.1] octan-3-one (15)

Yellow powder, mp $169.9-170.8{ }^{\circ} \mathrm{C}$; yield $77 \%,{ }^{1} \mathrm{H}$ NMR $\left(400 \mathrm{MHz}, \mathrm{CDCl}_{3}\right) \delta: 7.78$ (s, 2H, H-9,10), 7.74-7.22 (m, $6 \mathrm{H}$, thiophene- $\mathrm{H}), 4.48(\mathrm{~m}, 2 \mathrm{H}, \mathrm{H}-1,5), 2.63-2.61(\mathrm{~m}, 2 \mathrm{H}$, $\mathrm{H}-6,7$ ), 2.38 (s, 3H, H-8), 1.97-1.92 (m, 2H, H-6, 7). ${ }^{13} \mathrm{C}$ NMR $\left(100 \mathrm{MHz}, \mathrm{CDCl}_{3}\right) \delta: 187.4$ (s, C-3), 136.8 (s, C-2, 4), 136.6 (s, C-3', 3") 130.1 (d, C-9, 10), 129.1 (d, C- 2', $\left.2^{\prime \prime}\right), 128.3\left(\mathrm{~d}, \mathrm{C}-4^{\prime}, 4^{\prime \prime}\right), 126.2\left(\mathrm{~d}, \mathrm{C}-5^{\prime}, 5^{\prime \prime}\right), 61.2(\mathrm{~d}, \mathrm{C}-1$, 5), 36.0 (s, C-8), 30.0 (t, C-6, 7). IR (KBr) v: 3444, 2934, 1673, 1608, 1582, 1380, 1240, 1204, 1152, 1058, 928, $789 \mathrm{~cm}^{-1}$. ESIMS: $\mathrm{m} / z 328[\mathrm{M}+\mathrm{H}]^{+}$, HRESIMS: calc for $\mathrm{C}_{18} \mathrm{H}_{17} \mathrm{NOS}_{2}[\mathrm{M}+\mathrm{H}]^{+}$328.0824, found 328.0804.

\subsubsection{8-Methyl-2,4-bis(2'quinolylmethylene)-8-aza- bicyclo[3.2.1]octan-3-one (16)}

Yellow powder, mp 177.3-178.0 ${ }^{\circ} \mathrm{C}$; yield $76 \%,{ }^{1} \mathrm{H}$ NMR $\left(400 \mathrm{MHz}, \mathrm{CDCl}_{3}\right) \delta: 8.87(\mathrm{~d}, J=4.3 \mathrm{~Hz}, 2 \mathrm{H}$, quinolineH), 8.19 (s, 2H, H-9,10), 8.10-7.92 (m, 4H, quinoline-H), 7.69-7.49 (m, 4H, quinoline-H), $7.10(\mathrm{~d}, \mathrm{~J}=4.3 \mathrm{~Hz}, 2 \mathrm{H}$, quinoline-H), 4.04 (m, 2H, H-1, 5), 2.38 (m, 2H, H-6, 7), 2.18 (s, 3H, H-8), 1.93-1.88 (m, 2H, H-6, 7). ${ }^{13} \mathrm{C}$ NMR $\left(100 \mathrm{MHz}, \mathrm{CDCl}_{3}\right) \delta: 187.3$ (s, C-3), $149.6\left(\mathrm{~d}, \mathrm{C}-2^{\prime}, 2^{\prime \prime}\right)$, 148.2 (s, C-4', 4"), 142.4 (s, C-9', 9"), 140.7 (s, C-2, 4), 1301.7 (d, C-9, 10), 130.0 (d, C-7', 7"), 129.8 (d, C-8', 8"), 127.1 (d, C-6', 6"), 126.7 (s, C-10', 10") 124.5 (d, C-5', 5"), 120.7 (d, C-3', 3"), 61.0 (d, C-1, 5), 35.820 (s, C-8), 29.9 (t, C-6, 7). IR (KBr) v: 3441, 2943, 1683, 1612, 1580, 1563, 1503, 1417, 1388, 1204, 1166, 1061, 891, 845, $768 \mathrm{~cm}^{-1}$. ESIMS: $\mathrm{m} / z 418[\mathrm{M}+\mathrm{H}]^{+}$, HRESIMS: calc for $\mathrm{C}_{28} \mathrm{H}_{23} \mathrm{~N}_{3} \mathrm{O}[\mathrm{M}+\mathrm{H}]^{+}$418.1914, found 418.1932.

\section{Biological Test Methods}

The human tumor cell lines HL-60, SMMC-7721, A-549, MCF-7 and SW-480 were used, which were obtained from ATCC (Manassas, VA, USA). All cells were cultured in RPMI-1640 or DMEM medium (Hyclone, Logan, UT, USA), supplemented with $10 \%$ fetal bovine serum (Hyclone) and cultured with $95 \% \mathrm{O}_{2} / 5 \% \mathrm{CO}_{2}$ at $37{ }^{\circ} \mathrm{C}$. Cell viability was assessed by conducting colorimetric measurements of the amount of insoluble formazan formed in living cells based on the reduction of 3-(4,5-dimethylthiazol-2-yl)-5-(3-carboxymethoxyphenyl)-2-(4-sulfopheny)2H-tetrazolium (MTS, Sigma, St. Louis, MO, USA) [35]. The cells were seeded in a Matrigel coated 96-well black plate with a plating volume of $100 \mu \mathrm{L} /$ well at a density of $3000-15,000 /$ well, and incubated in $\mathrm{CO}_{2}$ incubator to adhere for $12 \mathrm{~h}$ before the tested drugs were added. Tested derivatives and positive drug were dissolved in dimethyl sulfoxide (DMSO) and extracted a plating volume of 20 $\mu \mathrm{L} /$ well in the Matrigel coated 96-well clear plate. With cisplatin and paclitaxel as positive controls, each tumor cell line was exposed to the test derivatives at $40 \mu \mathrm{M}$ concentrations in triplicate for $48 \mathrm{~h}$. After the incubation, MTS $(20 \mu \mathrm{L})$ was added to each well, and the incubation continued for $2-4 \mathrm{~h}$ at $37{ }^{\circ} \mathrm{C}$. The optical density of lysate was measured at $492 \mathrm{~nm}$ in a 96-well by Multiskan FC (Thermo Scientific, US). The inhibition rates expressed as $\overline{\mathrm{X}} \pm \mathrm{SD}(\mathrm{n}=3)$ were obtained. The $\mathrm{IC}_{50}$ value of each derivative was calculated by Reed and Muench's method [36].

Acknowledgement This work was supported by the Hundred Talents Program of the Chinese Academy of Sciences (CAS), the Youth Innovation Promotion Association, CAS and the Program of Yunling Scholarship.

\section{Compliance with Ethical Standards}

Conflict of interest The authors declare that there is no conflict of interest.

Open Access This article is distributed under the terms of the Creative Commons Attribution 4.0 International License (http:// creativecommons.org/licenses/by/4.0/), which permits unrestricted use, distribution, and reproduction in any medium, provided you give appropriate credit to the original author(s) and the source, provide a link to the Creative Commons license, and indicate if changes were made.

\section{References}

1. A. Silverstein, P. Mugenzi, J. Lule, A. Costas-Chavarri, J. Cancer Policy. 7, 1-3 (2016)

2. K. Strasser-Weippl, Y.C. Chavarri-Guerra, B.L. Bychkovsky, M. Debiasi, P.E. Liedke, E. Soto-Perez-de-Celis, D. Dizon, E. Cazap, G. de Lima Lopes, D. Touya, J.S. Nunes, J. Stlouis, C. Vail, A. Bukowski, P. Ramos-Elias, K. Unger-Saldaña, D.F. Brandao, M.E. Ferreyra, S. Luciani, A. Nogueira-Rodrigues, A.F. de Carvalho Calabrich, M.G. Del Carmen, J.A. Rauh-Hain, K. Schmeler, R. Sala, P. E. Goss. Lancet. Oncol. 16, 1405-1438 (2015)

3. J.B. Gibbs, Science 287, 1969-1973 (2000)

4. H. Varmus, Science 312, 1162-1165 (2006)

5. W.G. Kaelin, J. Clin. Invest. 104, 1503-1506 (1999)

6. H. Varmus, H.S. Kumar. Sci. Transl. Med. 5, 175cm2 (2013)

7. M. Gadzikowska, G. Grynkiewicz, Acta Pol. Pharm. 58, 481-492 (2001)

8. L. Melzig, A. Gavryushin, P. Knochel, Org. Lett. 26, 5529-5532 (2007)

9. K.C. Schmitt, J. Zhen, P. Kharkar, M. Mishra, N. Chen, A.K. Dutta, M.E.A. Reith, J. Neurochem. 107, 928-940 (2008)

10. R.H. Kline, S. Izenwasser, J.L. Katz, D.B. Joseph, W.D. Bowen, A.H. Newman, J. Med. Chem. 40, 851-857 (1997)

11. J.L. Katz, G.E. Agoston, K.L. Alling, R.H. Kline, M.J. Forster, W.L. Woolverton, T.A. Kopajtic, A.H. Newman, Psychopharmacology 154, 362-374 (2001)

12. G. Maksay, P. Nemes, Z. Vincze, T. Bíró, Bioorg. Med. Chem. 16, 2086-2092 (2008)

13. R.L. Papke, H.C. Schiff, B.A. Jack, N.A. Horenstein, Neurosci. Lett. 378, 140-144 (2005) 
14. K. Sun, Z.D. Guo, Int. J. Pharm. Res. 35, 87-91 (2008)

15. D.I. Lainé, H.B. Xie, N. Buffet, J.J. Foley, P. Buckley, E.F. Webb, K.L. Widdowson, M.R. Palovich, K.E. Belmonte. Bioorg. Med. Chem. Lett. 17, 6066-6069 (2007)

16. R. De Simone, L. Margarucci, V. De Feo, Pharmacologyonline. 1, 70-89 (2008)

17. J.W. Medley, M. Movassaghi, Chem. Commun. 49, 10775-10777 (2013)

18. Y.L. Hsu, P.L. Kuo, W.S. Tzeng, C.C. Lin, Food Chem. Toxicol. 44, 704-713 (2006)

19. D.K. Mahapatra, S.K. Bharti, V. Asati, Eur. J. Med. Chem. 98, 69-114 (2015)

20. A. Modzelewska, C. Pettit, G. Achanta, N.E. Davidson, P. Huang, S.R. Khan, Bioorgan. Med. Chem. 14, 3491-3495 (2006)

21. K.V. Sashidhara, A. Kumar, M. Kumar, J. Sarkar, S. Sinha, Bioorg. Med. Chem. 20, 7205-7211 (2010)

22. C.F. Jin, Y.J. Liang, H.W. He, L.W. Fu, Biomed. Pharmacother. 67, 215-217 (2013)

23. H.J. Zhang, Y. Qian, D.D. Zhu, X.G. Yang, H.L. Zhu, Eur. J. Med. Chem. 46, 4702-4708 (2011)

24. A. Kamal, G. Ramakrishna, P. Raju, A. Viswanath, M.J. Ramaiah, G. Balakishan, M. Pal-Bhadra, Bioorg. Med. Chem. Lett. 20, 4865-4869 (2010)

25. U. Das, J. Alcorn, A. Shrivastav, R.K. Sharma, E. De Clercq, J. Balzarini, J.R. Dimmock, Euro. J. Med. Chem. 42, 71-80 (2007)

26. A. Anantharaman, H. Hemachandran, S. Mohan, D.M. Ayyathan, T. Kumar, D, G.P. Doss C, R. Siva. J. Funct. Foods. 20, 346-357 (2016)
27. A. Anantharaman, B. Subramanian, R. Chandrasekaran, R. Seenivasan, R. Siva, Ind. Crop. Prod. 53, 167-186 (2014)

28. H.P. Chen, Z.Z. Zhao, Z.H. Li, Z.J. Dong, K. Wei, X. Bai, L. Zhang, C.N. Wen, T. Feng, J.K. Liu, ChemistryOpen. 5, 142-149 (2016)

29. L.D. Shao, Y.N. Wu, J. Xu, J. He, Y. Zhao, L.Y. Peng, Y. Li, Y.R. Yang, C.F. Xia, Q.S. Zhao, Nat. Prod. Bioprospect. 4, 181-188 (2014)

30. R. Chu, L.S. Wan, X.R. Peng, M.Y. Yu, Z.R. Zhang, L. Zhou, Z.R. Li, M.H. Qiu, Nat. Prod. Bioprospect. 6, 217-223 (2016)

31. G.R. Zheng, L.P. Dwoskin, A.G. Deaciuc, P.A. Crooks, Bioorg. Med. Chem. Lett. 15, 4463-4466 (2005)

32. D.I. Jung, C.S. Park, Y.H. Kim, D.H. Lee, Y.G. Lee, M. Park, S.K. Choi, Synth. Commun. 31, 3255-3263 (2001)

33. H.N. Pati, U. Das, S. Das, B. Bandy, E. De Clercq, J. Balzarini, M. Kawase, H. Sakagami, J.W. Quail, J.P. Stables, J.R. Dimmock, Eur. J. Med. Chem. 44, 54-62 (2009)

34. M. Suffness, J. Douros. Part A, in: V.T. De Vita Jr., H. Busch (Eds.), Methods in Cancer Research (Academic Press, New York, 1979) vol. 16 , p. 84

35. A. Monks, D. Scudiero, P. Skehan, R. Shoemaker, K. Paull, D. Vistica, C. Hose, J. Langley, P. Cronise, A. Vaigro-Wolff, J. Natl Cancer Inst. 83, 757-766 (1991)

36. L.J. Reed, H. Muench, Am. J. Hyg. 27, 493-497 (1938) 